\title{
LINER-like emission in red galaxies: evolutionary phase or recurring phenomenon?
}

\author{
Genevieve J. Graves ${ }^{1}$ \\ ${ }^{1}$ UCO/Lick Observatory, 1156 High Street, Santa Cruz, CA 95060, USA \\ email: graves@ucolick.org
}

\begin{abstract}
We present recent results showing that a large fraction of red sequence galaxies contain ionized gas with LINER-like optical emission line ratios. This emission is more frequently found in galaxies with lower central velocity dispersion $(\sigma)$ and these galaxies typically have younger mean ages than galaxies at the same $\sigma$ which do not host emission. We suggest that the presence of LINER-like emission may be determined by the quantity of interstellar material in these galaxies and may be associated with the recent accretion of a gas-rich satellite galaxy or alternatively with stellar mass loss that declines as the galaxy stellar population ages.
\end{abstract}

Keywords. galaxies: stellar content, galaxies: active, galaxies: bulges

\section{Introduction}

It is now firmly established at low redshift that the optical colors of galaxies form a bimodal distribution (e.g., Baldry et al. 2004). Recently, Yan et al. (2006) have demonstrated that the ratio between the $[\mathrm{OII}] \lambda 3727$ and $\mathrm{H} \alpha$ emission line equivalent widths $(\mathrm{EW}([\mathrm{OII}]) / \mathrm{EW}(\mathrm{H} \alpha))$ in galaxies also forms a bimodal distribution, which is strongly correlated with galaxy color (Yan et al. 2006). Galaxies with blue colors predominantly have low values of $\mathrm{EW}([\mathrm{OII}]) / \mathrm{EW}(\mathrm{H} \alpha)$, while galaxies with red colors (if they show detectable emission lines at all) have predominantly high values of $\mathrm{EW}([\mathrm{OII}]) / \mathrm{EW}(\mathrm{H} \alpha)$. Based on a set of emission line diagnostics using multiple optical emission lines, they showed that red galaxies with high $\mathrm{EW}([\mathrm{OII}]) / \mathrm{EW}(\mathrm{H} \alpha)$ have emission line ratios characteristic of low ionization nuclear emission-line regions (LINERs) and that these galaxies make up as much as $30 \%$ of the total number of red sequence galaxies. We shall hereafter refer to this emission as "LINER-like" emission because it displays emission line ratios characteristic of LINERs but the spatial distribution is unknown and may not in fact be "nuclear" as required by the term "LINER".

The existence of ionized gas in many local early type galaxies has been known for some time. Spectroscopic (e.g., Caldwell 1984, Phillips et al. 1986) and narrow-band imaging surveys (e.g., Goudfrooij et al. 1994, Macchetto et al. 1996) in the previous two decades detected emission from ionized gas in roughly $50 \%$ of local elliptical and S0 galaxies. The observed emission is typically extended over kpc scales and has emission line ratios consistent with LINER-like emission in the extended as well as the nuclear regions (Goudfrooij 1998). More recently, the SAURON project sample of E, S0, and Sa galaxies (de Zeeuw et al. 2002) has measured emission in early type galaxies with very high sensitivity, uncovering ionized gas emission in $\sim 75 \%$ of the 48 galaxies in their sample (Sarzi et al. 2006). In this sample as well, a large fraction of galaxies have extended emission with line ratios consistent with a LINER classification. 

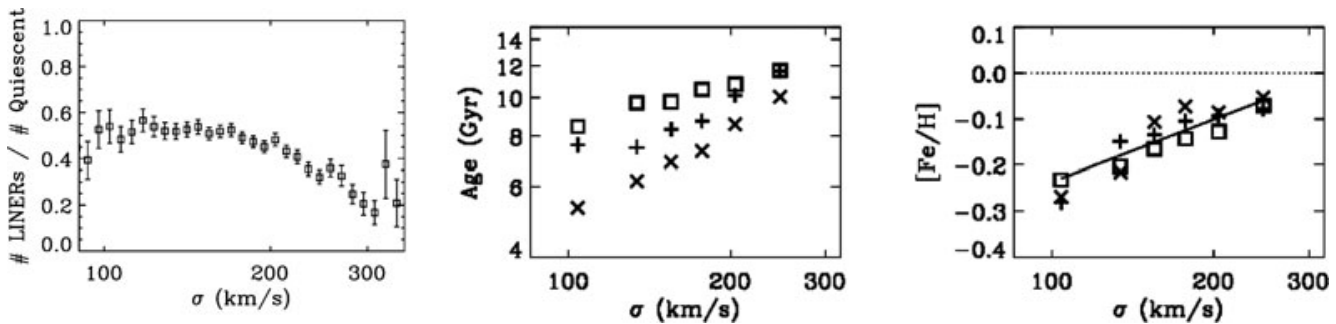

Figure 1. Left: Comparitive numbers of LINER-like and quiescent galaxies, as a function of $\sigma$. LINER-like galaxies are selected to have high values of $\mathrm{EW}([\mathrm{OII}]) / \mathrm{EW}(\mathrm{H} \alpha)$ and to have $\mathrm{EW}([\mathrm{OII}])>3 \AA$. Quiescent galaxies are selected to have $\mathrm{EW}([\mathrm{OII}])<3 \AA$ and $\mathrm{EW}(\mathrm{H} \alpha)<0.7 \AA$ (values corresponding to a $\sim 3 \sigma$ detection threshold at spectral $S / N \sim 20 \AA^{-1}$ ). The fraction of LINER-like galaxies is highest at lower values of $\sigma$ and declines in more massive galaxies. Error bars indicate Poisson statistics for each bin in $\sigma$. Center: Mean light-weighted ages meaured in stacked spectra as a function of $\sigma$. Open squares show quiescent galaxies, ' + ' symbols shows galaxies with weak LINER-like emission $(\mathrm{EW}([\mathrm{OII}])<5 \AA$ ), and ' $\mathrm{x}$ ' symbols show galaxies with strong LINER-like emission $(\mathrm{EW}([\mathrm{OII}])>5 \AA)$. As emission strength increases, galaxies have younger mean ages at fixed $\sigma$. Right: $[\mathrm{Fe} / \mathrm{H}]$ measured in stacked spectra as a function of $\sigma$ for quiescent galaxies and those with weak and strong LINER-like emission. LINER-like galaxies appear to have similar metallicities to their quiescent counterparts at fixed $\sigma$.

In this conference paper, we investigate in greater detail the stellar properties of the red sequence galaxies that host this LINER-like emission and compare them to their counterparts on the red sequence whose spectra are free of detectable emission lines.

\section{Stellar properties of galaxies with LINER-like emission}

The incidence with which ionized gas is detected in early type galaxies depends on the $S / N$ of the data used to make the measurement and may also vary with other galaxy properties. In Figure 1, we show the comparitive numbers of LINER-like galaxies and "quiescent" galaxies with no detectable emission, as a function of galaxy central velocity dispersion $(\sigma)$. These galaxies are selected from the SDSS (York et al. 2000) Main Galaxy Sample (Strauss et al. 2002) and only include galaxies with median spectral $S / N>20 \AA^{-1}$. It is clear from Figure 1 that LINER-like emission is most common in lower $\sigma$ galaxies and that the fraction of galaxies showing this emission declines as $\sigma$ increases. This may in fact indicate that this type of emission is more prevalent in S0 galaxies, which tend to be less massive systems, as opposed to E galaxies.

Graves et al. 2007 have recently analysed a sample of $\sim 6000$ SDSS galaxies within a narrow range of redshift $(0.06<z<0.08)$. The galaxies are chosen to have red optical colors and to have high values of $\mathrm{EW}([\mathrm{OII}]) / \mathrm{EW}(\mathrm{H} \alpha)$. These LINER-like galaxies are compared to a sample of quiescent galaxies with similar properties. Based on ensembles of stacked spectra, Graves et al. 2007 compute mean light-weighted ages, $[\mathrm{Fe} / \mathrm{H}]$, and abundance ratios for the LINER-like and quiescent galaxies using the stellar population models of Schiavon (2007).

Figure 1 shows the mean light-weighted ages and $[\mathrm{Fe} / \mathrm{H}]$ as a function of $\sigma$ for the LINER-like and quiescent galaxies (taken from Figures 12 and 14 of Graves et al. 2007). At fixed $\sigma$, the LINER-like galaxies are systematically younger than their quiescent counterparts, although their mean $[\mathrm{Fe} / \mathrm{H}]$ values are about the same. The abundances of other measurable elements $([\mathrm{Mg} / \mathrm{H}],[\mathrm{C} / \mathrm{H}],[\mathrm{N} / \mathrm{H}],[\mathrm{Ca} / \mathrm{H}]$, not shown) are also similar between quiescent and LINER-like galaxies, although they vary systematically with $\sigma$. These results indicate that LINER-like galaxies are either generally younger than 
quiescent galaxies at the same $\sigma$, or contain a sub-population of younger stars that makes their mean light-weighted age appear younger.

\section{A phase or a recurring phenomenon?}

Seeing as LINER-like galaxies are typically younger than their quiescent counterparts, this begs the question: is this LINER-like state a phase in galaxy evolution? It is not possible to answer this question definitively at this time, but there are several indications that this is not the case. If the LINER-like phase were indeed a transition phase, it has to be of long duration. At $\sigma=100 \mathrm{~km} \mathrm{~s}^{-1}$, LINER-like galaxies make up $\sim 1 / 3$ of the non-star-forming galaxy population (see Figure 1) and yet quiescent galaxies at this $\sigma$ have typical ages of $\sim 8$ Gyr (Figure 1), suggesting a duration of almost 3 Gyr for a transitional LINER-like phase.

Also, in Figure 1 the LINER-like galaxies at high $\sigma$ are older than the quiescent galaxies at low $\sigma$, thus it is clear that there is not a one-to-one correspondance between mean light-weighted galaxy age and the presence of LINER-like emission. Of course, if these galaxies have extended star formation histories, this may not fully disprove the hypothesis of a transitional phase in galaxy evolution. An alternative hypothesis is that the ionized gas in these galaxies is a recurring phenomenon, possibly associated with episodic periods of low-level star formation or "rejuvenation".

\section{Ionizing flux and the presence of gas}

In order to host LINER-like emission, a galaxy requires two things: a source of ionization and a supply of gas to be ionized. Many ionization mechanisms have been proposed to power LINER emission (see Filippenko 2003 for a review). One possibility we wish to highlight here is photoionization by post-asympototic giant branch (AGB) stars, as described in Binette et al. (1994). Macchetto et al. 1996 showed that the ionizing flux from post-AGB star predicted by Bruzual \& Charlot (1993) SSP models was enough to account for the observed $\mathrm{H} \alpha$ emission line luminosity in a sample of local early type galaxies. The $\mathrm{H} \alpha$ luminosities of the galaxies presented here are similar to those of Macchetto et al. 1996 for the same galaxy mass, thus this mechanism is adequate to account for the observed level of ionization in these systems.

An interesting implication of post-AGB stars as an ionizing source for LINERs is that every early type galaxy should have adequate ultraviolet (UV) flux to power low-level LINER-like emission. If this mechanism is feasible, the presence or absence of LINER-like emission becomes not a question of an ionization source, but one of gas supply. Perhaps galaxies with LINER-like emission are merely those with an adequate supply of gas to produce the observed emission.

There are several indications that LINER-like galaxies do indeed contain more interstellar material (ISM) than quiescent galaxies. Figure 1 shows that LINER-like galaxies are typically younger than their quiescent counterparts; we might therefore expect them to have slightly bluer optical colors (as predicted by stellar population models). In fact, the LINER-like galaxies are slightly redder than quiescent galaxies at the same $\sigma$ (Graves et al. 2007). This cannot be a metallicity effect, since the quiescent and LINER-like galaxies have almost the same total metallicity (see Figure 1). This strongly suggests that the LINER-like galaxies have higher levels of internal reddening than do quiescent galaxies and therefore contain more cold ISM, with an additional reddening of $E(B-V) \approx 0.1$ above the typical reddening of a quiescent galaxy at the same $\sigma$. In addition, the LINERlike galaxies show stronger absorption in the $\mathrm{NaD}$ feature at $5892 \AA$ than do quiescent 
galaxies, even though the absorption strengths are weaker in LINER-like galaxies for all other measured metal lines (Graves et al. 2007). In our own Galaxy, NaD is a resonant interstellar absorption feature, thus the observed excess in $\mathrm{NaD}$ absorption in LINERlike galaxies is likely another indication of a larger quantity of ISM. The measured excess of $\mathrm{NaD}$ is roughly consistent with the predicted $E(B-V) \approx 0.1$ determined from the observed galaxy colors (Graves et al. 2007). The indications that LINER-like galaxies contain more ISM are not conclusive, but they are at least consistent with the hypothesis that the difference between LINER-like and quiescent galaxies is the quantity of ISM, rather than the presence or absence of an ionizing source.

\section{Discussion}

We have shown in this conference paper that a substantial fraction of red sequence galaxies host LINER-like emission, and that these galaxies typically have younger mean light-weighted ages than quiescent galaxies at the same $\sigma$. We hypothesize here that every early type galaxy contains an adequate source of ionizing photons from post-AGB stars, so that the presence or absence of LINER-like emission is determined by the quantity of gas available for ionization. This hypothesis requires further investigation in order to be conclusively supported or disproven. If it is correct, then the connection between the presence of LINER-like emission and mean stellar population age requires an association between the current quantity of gas in the galaxy and its past star formation history. If the gas is of external origin, this could perhaps be explained by accretion of a small, gas-rich satellite along with its younger stellar population or else the accretion of gas followed by an associated epoch of star formation. If the gas is of internal origin through stellar mass loss, the observed age trend would require stellar mass loss rates to decline as the population ages, as proposed by Mathews (1989).

\section{Acknowledgements}

GG gratefully acknowledges support from NSF grant AST-05-07483.

\section{References}

Baldry, I. K., Glazebrook, K., Brinkmann, J., Ivezić, Z., Lupton, R. H., Nichol, R. C., \& Szalay, A. S. 2004, ApJ, 600, 681

Binette, L., Magris, C. G., Stasinska, G., \& Bruzual, A. G. 1994, A\&A, 292, 13

Bruzual, A. G. \& Charlot, S. 1993, ApJ, 405, 538

Caldwell, N. 1984, PASP, 96, 287

de Zeeuw, et al. 2002, MNRAS, 329, 513

Filippenko, A. V. 2003, ASPC, 290, 369 (astro-ph/030137)

Goudfrooij, P. 1999, in ASP Conf. Ser. 163: "Star Formation in Early-Type Galaxies", Eds. J. Cepa \& P. Carral, 163, 55 (astro-ph/9809057)

Goudfrooij, P., Hansen, L., Jørgensen, H. E., \& Nørgaard-Nielsen, H. U. 1994, A\& $\mathcal{E}$, 105, 341

Graves, G. J., Faber, S. M., Schiavon, R. P., \& Yan, R. 2007, ApJ accepted (astro-ph/0707.1523)

Macchetto, F., Pastoriza, M., Caon, N., Sparks, W. B., Giavalisco, M., Bender, R., \& Capaccioli, M. 1996, $A \& A, 120,463$

Mathews, W. G. 1989, AJ, 97, 42

Phillips, M. M., Jenkins, C. R., Dopita, M. A., Sadler, E. M., \& Binette, L. 1986, AJ, 91, 1062

Sarzi, M., et al. 2006, MNRAS, 366, 1151

Schiavon, R. P. 2007, ApJS, 171, 146

Strauss, M. A., et al. 2002, AJ, 124, 1810

Yan, R., Newman, J. A., Faber, S. M., Konidaris, N., Koo, D., \& Davis, M. 2006, ApJ, 648, 281

York, D. G., et al. 2000, AJ, 120, 1579 